\title{
Deformable Bayesian Networks for Data Clustering and Fusion
}

\author{
Kittipat Kampa ${ }^{a}$, Jose C. Principe ${ }^{a}$, J. Tory $\mathrm{Cobb}^{b}$, and Anand Rangarajan ${ }^{c}$ \\ ${ }^{a}$ Department of Electrical and Computer Engineering, University of Florida, Gainesville, FL, \\ 32611, USA; \\ ${ }^{b}$ Naval Surface Warfare Center Panama City Division, Panama City, FL 32407, USA; \\ ${ }^{c}$ Department of Computer and Information Science and Engineering, University of Florida, \\ Gainesville, FL, 32611, USA
}

\begin{abstract}
In this work, we propose DEformable BAyesian Networks (DEBAN), a probabilistic graphical model framework where model selection and statistical inference can be viewed as two key ingredients in the same iterative process. While this concept has shown successful results in computer vision community, ${ }^{1-4}$ our proposed approach generalizes the concept such that it is applicable to any data type. Our goal is to infer the optimal structure/model to fit the given observations. The optimal structure conveys an automatic way to find not only the number of clusters in the data set, but also the multiscale graph structure illustrating the dependence relationship among the variables in the network. Finally, the marginal posterior distribution at each root node is regarded as the fused information of its corresponding observations, and the most probable state can be found from the maximum a posteriori (MAP) solution with the uncertainty of the estimate in the form of a probability distribution which is desired for a variety of applications.
\end{abstract}

Keywords: Bayesian networks, deformable structure, Gaussian mixture model, unsupervised, clustering algorithm

\section{INTRODUCTION}

Probabilistic graphical models have been successfully applied to many real-world applications. As being a marriage between graph theory and probability theory, a probabilistic graphical model provides intuitive ways to represent data that are observed partially or with some uncertainties due to noise in the system. In this work, we propose a novel graphical model framework to data clustering and fusion which currently remain challenging problems in many applications.

In order to exploit a graphical model to an application, there are 2 main issues to consider: 1) the graphical model structure learning problem and 2) the inference problem. The former problem involves how to come up with a good structure to associate the random variables such that the graphical model best fits the observed data. This problem is, in fact, equivalent to the model selection problem in graphical models which has been a large and active area for research for decades. ${ }^{5-8}$ The latter problem deals with inferring probability distribution/mass function (pdf/pmf) at unobserved (hidden) nodes in the model. At this moment, advances in statistical machine learning and related areas provide many approaches to solve this problem efficiently and systematically.

Both the structure learning problem and the inference problem are so challenging that most of the time they are treated separately and independently even though in many applications they should be treated as dependent processes. In a particular application, the structure is usually assumed known to a human operator and is fixed throughout the procedure, leaving only the corresponding inference problem to be solved. Unfortunately, in a system where there are many variables or the variables are non-stationary, the fixed structure graphical model may be extremely inaccurate in the resulting prediction. These potential problems emphasize the need for a graphical model framework where structure learning and inference are treated dependently.

Further author information: (Send correspondence to Kittipat Kampa)

Kittipat Kampa: E-mail: kittipat@gmail.com, Telephone: 13523922682

8017 - 25 V. 1 (p.1 of 15) / Color: No / Format: Letter / Date: 2011-04-10 03:42:58 PM

SPIE USE: DB Check, Prod Check, Notes: 
In this work, we propose DEformable BAyesian Networks (DEBAN), a probabilistic graphical model framework where model selection and statistical inference can be viewed as two principal ingredients in the same iterative process. While this concept has shown successful results in computer vision community, ${ }^{1-4}$ our proposed approach generalizes the concept such to be applicable to any data type. Our framework is based on directed acyclic graphical models, a.k.a Bayesian networks. In the Bayesian networks we describe here, there are two types of random variables; hidden and observed denoted by $X$ and $Y$ respectively. The underlying graph structure $Z$ encodes the dependence relationship among random variables $X$ and $Y$, each of which is represented by a node in the Bayesian network structure. In order to incorporate the behavior of the data set at various scales and to preserve long range correlations between a pair of nodes, the structure $Z$ is restricted a to hierarchical tree structure like the tree-structure Bayesian Networks (TSBN). ${ }^{9}$ The tree structure enables not only the exact inference algorithm, but also the efficient computational time for the hidden nodes. Each directed edge of the graph structure $Z$ represents the conditional probability table (cpt) of the child node (node attached to the arrow) given its corresponding parent (node at the other end of the arrow). We restrict our attention to generative models for all the cpts in the network.

Unlike a TSBN, the graph structure in our proposed model can adapt according to the log-likelihood of the structure given the distribution at each hidden node. In Dynamic Tree (DT) Bayesian networks, ${ }^{1-3}$ the proposed concept has been demonstrated to alleviate the "blocky effect" caused by fixed graph structure in TSBN and to improve the accuracy of image classification significantly. While DT formulation assumes that the observed variables are discrete, DEBAN makes a clear separation between the observed variables and hidden variables which makes it more generalized. Our work also provides a special case where the state-transition distribution $p\left(X_{j}=x_{j} \mid X_{i}=x_{i}\right)$ is multinomial and the state-observation distribution $p\left(Y_{e}=y_{e} \mid X_{i}=x_{i}\right)$ is Gaussian distributed which is applicable to many applications and easy to understand. The structure $Z$ is treated as a random variable in the system. DEBAN can be seen as an iterative process composed of two alternating algorithms:

1. Given the likelihood of the structure $Z$ and the conditional probabilities in the network, the posterior marginal distribution at each node in the network is calculated using the sum-product algorithm. ${ }^{10}$

2. Given the posterior marginal distribution at each node, we adapt the structure $Z$ such that the cost function is increased.

The two processes are performed alternately until the algorithm converges. Using this process, we can infer the maximum a posteriori (MAP) structure $Z^{*}$ that maximizes the posterior $p(Z \mid Y)$. Two criteria decide the optimal structure $Z^{*}: 1$ ) the number of clusters in the data set, and 2) the likelihood that an observation is generated from a particular cluster. The number of clusters can be determined from the number of the root nodes in the final graph structure $Z^{*}$ and the membership assigned to each observation is found from the optimal structure and the MAP state of its corresponding parent node.

\section{DEFORMABLE BAYESIAN NETWORKS (DEBANS)}

In this section, before discussing our proposed framework we first present an overview of Bayesian networks. Next the probabilistic model of DEBAN is mathematically described, followed by its special case where the state-observation probability distribution of each class is multivariate Gaussian distributed.

\subsection{Bayesian Networks}

In a Bayesian network (BN) graphical model, the joint probability of a set of random variables (or nodes) is defined by the connections of the graph which in turn define the conditional probability relationships amongst the nodes. In Fig. 1 a set of random variables $A, B, C, D$ is connected as shown. Directed links from parent node to child nodes define the probability state of the children conditioned on the parents. For example, the arrow from node $A$ to node $C$ in Fig. 1 denotes the conditional probability $P(C \mid A)$. Root nodes or nodes which do not have a parent are defined through a prior probability. Through this notation the joint probability of the graph in Fig. 1 can be factored as

$$
P(A, B, C, D)=P(A) P(B \mid A) P(C \mid A) P(D \mid B, C) .
$$

\section{7 - 25 V. 1 (p.2 of 15) / Color: No / Format: Letter / Date: 2011-04-10 03:42:58 PM}




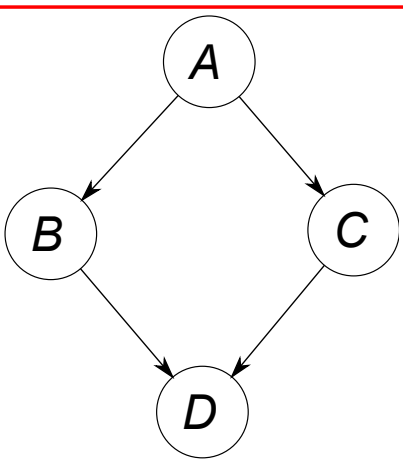

Figure 1. Bayesian network graphical model. Nodes $A, B, C$, and $D$ are random variables, and links between the nodes define conditional probability relationships between parent and child nodes.

Normally there are two types of variables or nodes in a graphical model; 1) observed or evidential variables and 2) hidden or unobserved variables. Most of the time, our goal is to infer the probability distribution of a subset of hidden nodes given the evidential nodes via posterior distribution derived from Bayes' rule and marginalizing out other hidden nodes that are out of our interest. For example, to infer the probability of the states of random variable $C$ when the values at nodes $B=b$ and $D=d$ in Fig. 1 requires that we sum over the probability mass function of variable $A$ in the numerator; and $A$ and $C$ in the denominator of (1). This expression (i.e. the posterior) can be written according to Bayes' rule as

$$
p(c \mid b, d)=\frac{\sum_{a \in \mathcal{A}} p(a) p(b \mid a) p(c \mid a) p(d \mid b, c)}{\sum_{(a, c) \in \mathcal{A} \times \mathcal{C}} p(a) p(b \mid a) p(c \mid a) p(d \mid b, c)}
$$

The computational complexity of marginalization increases as the number of nodes, states and links increases in the BN. In most inference cases, the probability mass/distribution function at each node in the BN needs to be updated after the introduction of new evidence into the network. When the number of hidden nodes to be marginalized is large, the normalization factor (i.e. the denominator in Eq. (2)) can be computationally intractable. Fortunately, there are several algorithms that can make marginalization tractable. For tree-structured networks, i.e. each child node has only one parent, the marginalization or belief update at each node can be carried out exactly (i.e. exact inference) using a fast sum-product algorithm known as belief propagation (BP) or Pearl's message passing. ${ }^{10}$ BP gives exact inference for a tree-structured network and gives approximate inference for a network containing cycles in which case it is called loopy belief propagation (LBP). ${ }^{11} \mathrm{BP}$ greatly reduces computation time and provides a standard programming framework to accomplish the marginalization over the entire network. In this work, we restrict the framework to a tree-structured network and use BP due to the computational advantages.

\subsection{Probabilistic Model of Generalized DEBAN}

Let $X=\left\{x_{h}\right\}_{h \in \mathcal{H}}$ denote a set of hidden nodes, where $\mathcal{H}$ is the set of indices of the hidden nodes; and let $Y=\left\{y_{e}\right\}_{e \in \mathcal{E}}$ denote a set of observed nodes, where $\mathcal{E}$ is the set of indices of the observed nodes. The number of nodes in the network will be $N=|\mathcal{H}|+|\mathcal{E}|$. Moreover, let $\mathcal{H}^{l}$ denote a set of indices of nodes in hidden layer $l \in\{1,2, \ldots, L-1\}$ where $L-1$ is the root (top-most) layer as show in Fig. 2. We set the index for each node in the network in ascending order from root to leaf. In other words, the index of a parent node is smaller than that of its children. A model with a fixed structure $Z$ is defined by the joint distribution $p(X, Y \mid Z, \Theta)$. Since DEBAN is a directed acyclic graph (DAG), the joint factorizes over the nodes, i.e. $p(X, Y \mid Z, \Theta)=\prod_{j} p\left(u_{j} \mid p a_{j}, \theta_{j}, Z\right)$, where $u_{j} \in X \cup Y, p a_{j}$ is the set of parents of $u_{j}$, and $\theta_{j} \in \theta$ parameterizes the edges (conditional probability) pointing toward the child node $u_{j}$. The structure of the model is determined by the variable $Z=\left\{z_{j i}\right\}_{(j, i) \in \mathcal{A}}$, where all possible connections are determined by $\mathcal{A}=\left\{\mathcal{E} \times \mathcal{H}^{1}\right\} \cup\left\{\mathcal{H}^{l} \times \mathcal{H}^{l+1}\right\}_{l=1}^{L-1}$, and $z_{j i}=1$ when the child node $u_{j}$ is connected to the parent $u_{i}$, otherwise $z_{j i}=0$. Normally, $Z$ will be written in the form of an $N \times N$ 


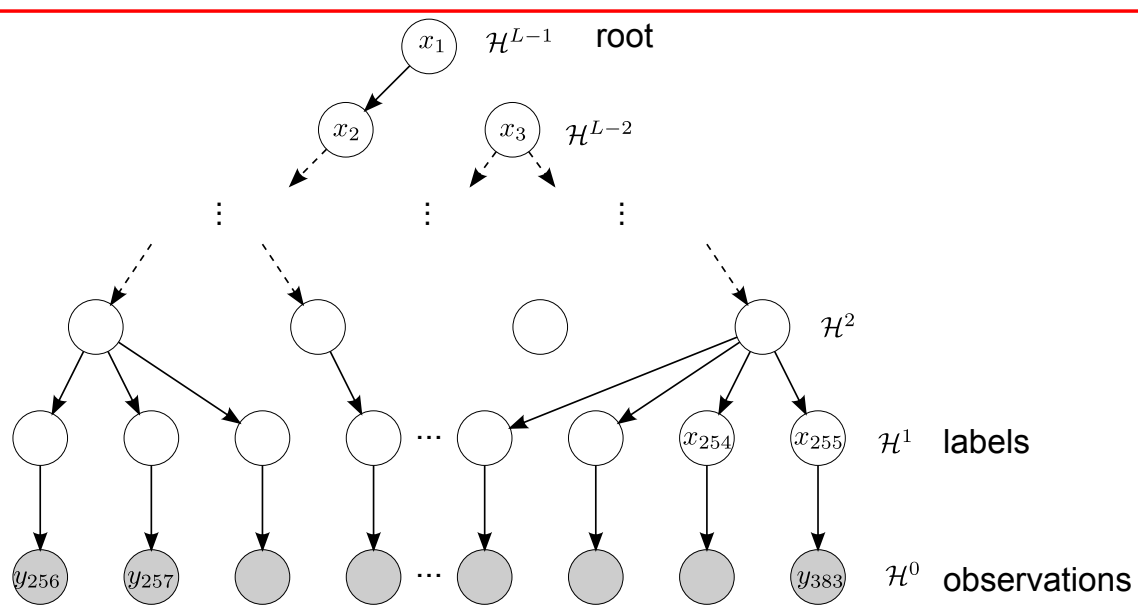

Figure 2. A graphical overview of DEformable BAyesian Networks (DEBAN)

matrix whose element in $j^{\text {th }}$ row and $i^{\text {th }}$ column is $z_{j i}$. The joint probability $P(X, Y, Z \mid \Theta)$ defines all possible state configurations and all possible structures of the DEBAN and can be factorized as

$$
P(X, Y, Z \mid \Theta)=P(X, Y \mid Z, \theta) P(Z \mid \pi),
$$

where $\Theta=\{\theta, \pi\} ; \theta_{j i} \in \theta$ denote parameters of the edge from $u_{i}$ toward $u_{j} ; \pi_{j i} \in \pi$ denotes the probability that the child node $u_{j}$ is connected to the parent node $u_{i}$, i.e. $p\left(z_{j i}=1\right)=\pi_{j i}$. In this work, we restrict our attention to a tree-structured architecture for $Z$ because of its computational efficiency for inference. The joint probability given a structure $Z$ (a.k.a structure likelihood) can be written as:

$$
\begin{aligned}
p(X, Y \mid Z, \theta)= & \prod_{e \in \mathcal{E}} \prod_{i \in \mathcal{H}^{1}} p\left(y_{e} \mid x_{i}, \theta_{e i}\right)^{z_{e i}} \\
& \cdot \prod_{l=1}^{L-1}\left(\prod_{j \in \mathcal{H}^{l}} \prod_{i \in\left\{0, \mathcal{H}^{l+1}\right\}} p\left(x_{j} \mid x_{i}, \theta_{j i}\right)^{z_{j i}}\right) .
\end{aligned}
$$

Note that the form of structure likelihood does not depend on the functional form of $Z$ at all. Also note that we have 0 in the set of parent nodes $\left\{0, \mathcal{H}^{l+1}\right\}$ indicating that any node in $\mathcal{H}^{l}$ connecting to the 0 -node becomes a root node and $\mathcal{H}^{L}$ is an empty set, so root nodes in layer $\mathcal{H}^{L-1}$ are automatically connected to 0 .

\subsection{A Special Case of DEBAN}

In this subsection, we restrict our attention to a simple special case of DEBAN where the state-transition distribution $p\left(x_{j} \mid x_{i}, \theta_{j i}\right)$ is multinomial and the state-observation distribution $p\left(y_{e} \mid x_{i}\right)$ is Gaussian-distributed which is applicable to many applications and is easy to understand. In this paper, the structure is updated using a heuristic method, which will be discussed in later sections. For simplicity, we assume for the moment that the structure $Z$ is fixed and the value of $z_{j i}$ is known. From this point forward, we will use the terminology state-transition distribution and conditional probability table $(\mathrm{cpt})$ interchangeably. Each hidden node $x_{i}$ can take a vector value from a set of states* $\mathcal{C}=\left\{1_{1}, \ldots, 1_{C}\right\}$ where $1_{c}$ is a $C \times 1$ column vector with 1 only at the position $c$ and 0 elsewhere. Hence, the cpt is given by:

$$
p\left(x_{j} \mid x_{i}, \theta_{j i}\right)=\prod_{v=1}^{C} \prod_{u=1}^{C} \phi_{j i v u}^{x_{j v} x_{i u}},
$$

where $\phi_{j i v u}$ denotes the value of the state-transition probability $p\left(x_{j v}=1 \mid x_{i u}=1, \phi_{j i}, j\right)$ and $x_{j u}$ denotes the $u^{\text {th }}$ row of vector $x_{j}$. Assume that the $\operatorname{cpt} \phi$ is shared among nodes in the same level, i.e. $\phi_{j i v u}=\phi_{j^{\prime} i^{\prime} v u}=\phi_{l v u}$

*In this paper, the notion of a state can mean the same thing as class. That is why we use $C$ to represent the number of states for hidden variables. 
where $j, j^{\prime} \in \mathcal{H}^{l}$ and $i, i^{\prime} \in \mathcal{H}^{l+1}$ for $l \in\{1, \ldots, L-1\} ; v, u \in\{1, \ldots, C\}$. Hence let $\phi=\left\{\phi_{l v u}\right\}_{l \in\{1, \ldots, L-1\}, v \times u \in\{\mathcal{C} \times \mathcal{C}\}}$ collectively denote the cpt of the model which is given by

$$
p\left(x_{j} \mid x_{i}, \phi, l\right)=\prod_{v=1}^{C} \prod_{u=1}^{C} \phi_{l v u}^{x_{j v} x_{i u}} .
$$

Each $y_{e}$ is a continuous-valued random variable node which can take any $D \times 1$ real-valued vector. In this work we assume that the number of the states is known as $C$, and a class can be represented by one multivariate Gaussian, thus, the state-observation probability distribution $p\left(y_{e} \mid x_{i}\right)$ is given by

$$
p\left(y_{e} \mid x_{i}\right)=\prod_{c=1}^{C} \mathcal{N}\left(y_{e} \mid \mu_{c}, \Lambda_{c}^{-1}\right)^{x_{i c}}
$$

where $\mathcal{N}\left(y_{e} \mid \mu_{c}, \Lambda_{c}^{-1}\right)=\frac{\left|\Lambda_{c}\right|^{1 / 2}}{(2 \pi)^{D / 2}} \exp \left[-\frac{1}{2}\left(y_{e}-\mu_{c}\right)^{\top} \Lambda_{c}\left(y_{e}-\mu_{c}\right)\right] ; \mu_{c}$ and $\Lambda_{c}$ are respectively $D \times 1$ mean vector and $D \times D$ precision matrix for class $c$. Each $x_{i c}$ can only take the value of 1 or 0 , and $\sum_{c=1}^{C} x_{i c}=1$. From all above, the joint distribution can be written as

$$
\begin{aligned}
p(X, Y \mid Z, \theta)= & \prod_{e \in \mathcal{E}} \prod_{i \in \mathcal{H}^{1}} \prod_{c=1}^{C} \mathcal{N}\left(y_{e} \mid \mu_{c}, \Lambda_{c}^{-1}\right)^{x_{i c} z_{e i}} \\
& \cdot \prod_{l=1}^{L-1}\left(\prod_{j \in \mathcal{H}^{l}} \prod_{i \in\left\{0, \mathcal{H}^{l+1}\right\}} \prod_{v=1}^{C} \prod_{u=1}^{C} \phi_{l v u}^{x_{j v} x_{i u} z_{j i}}\right)
\end{aligned}
$$

and the corresponding log-likelihood is

$$
\begin{aligned}
\log p(X, Y \mid Z, \theta)= & \sum_{e \in \mathcal{E}} \sum_{i \in \mathcal{H}^{1}} z_{e i} \sum_{c=1}^{C} x_{i c} \log \mathcal{N}\left(y_{e} \mid \mu_{c}, \Lambda_{c}^{-1}\right) \\
& +\sum_{l=1}^{L-1}\left(\sum_{j \in \mathcal{H}^{l}} \sum_{i \in\left\{0, \mathcal{H}^{l+1}\right\}} z_{j i} \sum_{v=1}^{C} \sum_{u=1}^{C} x_{j v} x_{i u} \log \phi_{l v u}\right)
\end{aligned}
$$

\section{LEARNING PARAMETERS OF DEBAN}

Since the variables $x$ in the log-likelihood in Eq. 3 are not observed, in order to learn the parameters $\phi, \mu_{c}$ and $\Lambda_{c}$ we use the EM algorithm. ${ }^{12,13}$ In this section, we derive for DEBAN a set of EM update equations obtained from the Expectation step (E-step) and Maximization step (M-step) respectively.

\subsection{E-step}

In this step, we calculate the expected value, denoted by the symbol $\langle\cdot\rangle$, of the complete-data log-likelihood $\log p(X, Y \mid Z, \theta)$ with respect to unknown data $X$ given the observed data $Y$. That is, we define

$$
\begin{aligned}
\mathcal{F}\left(\theta ; \theta^{t-1}\right) \triangleq & \langle\log p(X, Y \mid Z, \theta)\rangle_{p\left(X \mid Y, Z, \theta^{t-1}\right)} \\
= & \sum_{e \in \mathcal{E}} \sum_{i \in \mathcal{H}^{1}} z_{e i} \sum_{c=1}^{C}\left\langle x_{i c}\right\rangle_{p\left(X \mid Y, Z, \theta^{t-1}\right)}\left(\frac{1}{2} \log \left|\Lambda_{c}\right|-\frac{D}{2} \log 2 \pi-\frac{1}{2}\left(y_{e}-\mu_{c}\right)^{\top} \Lambda_{c}\left(y_{e}-\mu_{c}\right)\right) \\
& +\sum_{l=1}^{L-1}\left(\sum_{j \in \mathcal{H}^{l}} \sum_{i \in\left\{0, \mathcal{H}^{l+1}\right\}} z_{j i} \sum_{v=1}^{C} \sum_{u=1}^{C}\left\langle x_{j v} x_{i u}\right\rangle_{p\left(X \mid Y, Z, \theta^{t-1}\right)} \log \phi_{l v u}\right) .
\end{aligned}
$$


The expectation terms can be calculated from

$$
\begin{aligned}
\left\langle x_{i c}\right\rangle_{p\left(X \mid Y, Z, \theta^{t-1}\right)} & =\sum_{X} x_{i c} p\left(X \mid Y, Z, \theta^{t-1}\right) \\
& =\sum_{x_{i}} x_{i c} p\left(x_{i} \mid Y, Z, \theta^{t-1}\right) \\
& =p\left(x_{i c}=1 \mid Y, Z, \theta^{t-1}\right)
\end{aligned}
$$

and

$$
\left\langle x_{j v} x_{i u}\right\rangle_{p\left(X \mid Y, Z, \theta^{t-1}\right)}=p\left(x_{j v}=1, x_{i u}=1 \mid Y, Z, \theta^{t-1}\right) .
$$

Both expectation terms can be efficiently calculated using the sum-product algorithm. ${ }^{14}$

\subsection{M-step}

In this step, we equate to zero the derivative of the cost function $\mathcal{F}\left(\theta ; \theta^{t-1}\right)$ with respect to each parameter:

Learning the parameter $\mu_{c}$ :

$$
\frac{\partial \mathcal{F}\left(\theta, \theta^{t-1}\right)}{\partial \mu_{c}}=\sum_{e \in \mathcal{E}} \sum_{i \in \mathcal{H}^{1}} z_{e i}\left\langle x_{i c}\right\rangle_{p\left(X \mid Y, Z, \theta^{t-1}\right)} \Lambda_{c}\left(y_{e}-\mu_{c}\right)
$$

and equating the derivative to 0 , we have

$$
\mu_{c}=\frac{\sum_{e \in \mathcal{E}} \sum_{i \in \mathcal{H}^{1}} z_{e i}\left\langle x_{i c}\right\rangle_{p\left(X \mid Y, Z, \theta^{t-1}\right)} y_{e}}{\sum_{e \in \mathcal{E}} \sum_{i \in \mathcal{H}^{1}} z_{e i}\left\langle x_{i c}\right\rangle_{p\left(X \mid Y, Z, \theta^{t-1}\right)}}
$$

Learning the parameter $\Lambda_{c}$ :

$$
\frac{\partial \mathcal{F}\left(\theta, \theta^{t-1}\right)}{\partial \Lambda_{c}}=\sum_{e \in \mathcal{E}} \sum_{i \in \mathcal{H}^{1}} z_{e i}\left\langle x_{i c}\right\rangle_{p\left(X \mid Y, Z, \theta^{t-1}\right)} \frac{1}{2}\left[\Lambda_{c}^{-1}-\left(y_{e}-\mu_{c}\right)\left(y_{e}-\mu_{c}\right)^{\top}\right]
$$

and equating the derivative to 0 , we have

$$
\Lambda_{c}^{-1}=\frac{\sum_{e \in \mathcal{E}} \sum_{i \in \mathcal{H}^{1}} z_{e i}\left\langle x_{i c}\right\rangle_{p\left(X \mid Y, Z, \theta^{t-1}\right)}\left(y_{e}-\mu_{c}\right)\left(y_{e}-\mu_{c}\right)^{\top}}{\left.\sum_{e \in \mathcal{E}} \sum_{i \in \mathcal{H}^{1}} z_{e i}\left\langle x_{i c}\right\rangle_{p\left(X \mid Y, Z, \theta^{t-1}\right.}\right)} .
$$

Learning the parameter $\phi$

Here we will learn each $\phi_{l v u}$ under the constraint that $\sum_{v} \phi_{l v u}=1$, so the Lagrange multiplier term $-\lambda\left(\sum_{v} \phi_{l v u}-\right.$ 1) will be incorporated into the log-likelihood term

$$
\frac{\partial \mathcal{F}\left(\theta, \theta^{t-1}\right)}{\partial \phi_{l v u}}=\sum_{j \in \mathcal{H}^{l}} \sum_{i \in\left\{0, \mathcal{H}^{l+1}\right\}} z_{j i}\left\langle x_{j v} x_{i u}\right\rangle_{p\left(X \mid Y, Z, \theta^{t-1}\right)} \frac{1}{\phi_{l v u}}-\lambda .
$$

Equating the above derivative to 0 , we have

$$
\phi_{l v u}=\frac{\sum_{j \in \mathcal{H}^{l}} \sum_{i \in\left\{0, \mathcal{H}^{l+1}\right\}} z_{j i}\left\langle x_{j v} x_{i u}\right\rangle_{p\left(X \mid Y, Z, \theta^{t-1}\right)}}{\lambda},
$$

where $\lambda=\sum_{v} \sum_{j \in \mathcal{H}^{l}} \sum_{i \in\left\{0, \mathcal{H}^{l+1}\right\}} z_{j i}\left\langle x_{j v} x_{i u}\right\rangle_{p\left(X \mid Y, Z, \theta^{t-1}\right)}$. It is more convenient to write

$$
\phi_{l v u}=\frac{\hat{\phi}_{l v u}}{\sum_{v^{\prime}} \hat{\phi}_{l v^{\prime} u}}
$$

where $\hat{\phi}_{l v u}=\sum_{j \in \mathcal{H}^{l}} \sum_{i \in\left\{0, \mathcal{H}^{l+1}\right\}} z_{j i}\left\langle x_{j v} x_{i u}\right\rangle_{p\left(X \mid Y, Z, \theta^{t-1}\right)}$ denotes the unnormalized class cpt. 


\subsection{Initializing $\phi$}

One problem for the EM-algorithm is local minima, so a good initialization usually contributes to a good final solution. We propose $k$ nearest neighbor density walk (KDW) algorithm, a fast and intuitive approach to calculating the initial cpt $\phi$. First, the user has to pick parameters: the length of density walk $L$, number $k$ for $k$-nearest neighbor $(k$-nn) and the number of samples $S$. Next, a $C$-component Gaussian mixture model (GMM) to the observation $Y$ and make a simple $C$-class Bayes classifier from this GMM. This classifier will be used very often in the following steps. After that, randomly pick a starting point $y_{1}$ from the observation $Y$, then calculate $k$-nn of $y_{1}$ and randomly pick one of them, the selected point is called $y_{2}$. Repeat this process until the sample $y_{L}$ is obtained. This creates a series of points generated from a random-walk process guided by the regions of high density - this is called a $k$-nn density walk of size $L$ - as a result, the first density walk $R^{1}$ is made. The density walk will be generated $S$ times. Let $y_{l}^{s}$ denote the $l^{\text {th }}$ sample of the walk $R^{s}$. As $S$ is getting bigger, the cpt will be better represented. Next, the walks $R^{1}, \ldots, R^{S}$ are used to produce the initial cpt, $\phi^{0}$, for the whole network. The element at $r^{t h}$ row and $c^{t h}$ column is denoted by $\phi_{r c}^{0}=\frac{\hat{\phi}_{r c}^{0}}{\sum_{r^{\prime}} \hat{\phi}_{r^{\prime} c}^{0}}$ where $\hat{\phi}_{r c}^{0}=\sum_{s=1}^{S} \delta_{c}\left(M A P\left(y_{1}^{s}\right)\right) \sum_{l=2}^{L} \delta_{r}\left(M A P\left(y_{l}^{s}\right)\right), M A P\left(y_{l}^{s}\right)$ denotes the maximum a posteriori state of $y_{l}^{s}$ classified by the GMM earlier and $\delta_{r}(\cdot)$ denotes the Kronecker delta, i.e. $\delta_{r}(x)=1$ when $x=r$, and 0 otherwise.

The elements in the cpt indicate the strength of the connections among the class. The cpt built from the KDW algorithm captures the relations among the Gaussian distribution components and proposes the topology of the cluster in a probabilistic way. Different ways of making the cpt would capture different meanings of the relations among the classes. There is a very strong connection to be discussed further between parameters $k$ and $L$ with kernel width in non-parametric pdf estimation.

\subsection{Kernel Perspective of KDW}

Like in non-parametric pdf estimation, choosing parameters $k$ and $L$ is crucial in this application. There is a strong connection between the physical meaning of $k$ and $L$ with the kernel size in non-parametric pdf estimation. As mentioned earlier in this section, the cpt is supposed to capture the relationships among the Gaussian components, but such a relationship can be subjective and data set-dependent, so in order to make an appropriate choice, we need to fully understand the behavior of the parameters. One way to understand it is to compare with those of non-parametric pdf estimation which share similar behavior.

When the number of nearest neighbor $k$ is big, the radius of candidate points is big, so the density walk tends to have greater chance than small $k$ to take a long walk. Thus, the chance that the density walk would pass multiple components would be high, however, the chance that the path would include undesired clusters is high too, so picking an appropriate size of $k$ is very important. This behavior is equivalent to having a big kernel size in non-parametric pdf estimation. Furthermore, $L$ has very similar behavior to $k$ and they both work to complement each other. In an extreme case when $k$ and $L$ are on the order of the number of points in the space, each column in the cpt shifts toward the prior probability.

A numerical experiment is conducted on the "frame2" data set containing 400 samples of 2-dimensional vectors. The values of $k$ are 3, 10 and 20 from left to right. The values of $L$ are 30, 100 and 200 from top to bottom. $C=4$ was assumed to be the number of components in the GMM. The $k$-nn density walk map of $S=3$ is shown in Fig. 3. The cpts are shown in Fig. 4 for $S=50$ which was used in this experiment. Fig. 3 supports the claim that when $k$ and $L$ increase, the chance that the density walk skips between several clusters increases as well, and each column in the cpt converges to the prior probability as shown in Fig. 4 (g-i).

\section{INFERENCE IN DEBAN}

The goal of a DEBAN is to search for the optimal structure $Z^{*}$ that can explain the observations best, that is the structure that maximizes the posterior marginal $P(Z \mid Y, \Theta) .^{2}$ From this point on, it will be understood that the probabilities are given with the model parameters $\Theta$, but we will not write it explicitly. The posterior of the structure can be calculated from

$$
p(Z \mid Y)=\frac{\sum_{X} p(X, Y, Z)}{\sum_{X, Z} p(X, Y, Z)} .
$$

\section{7 - 25 V. 1 (p.7 of 15) / Color: No / Format: Letter / Date: 2011-04-10 03:42:58 PM}




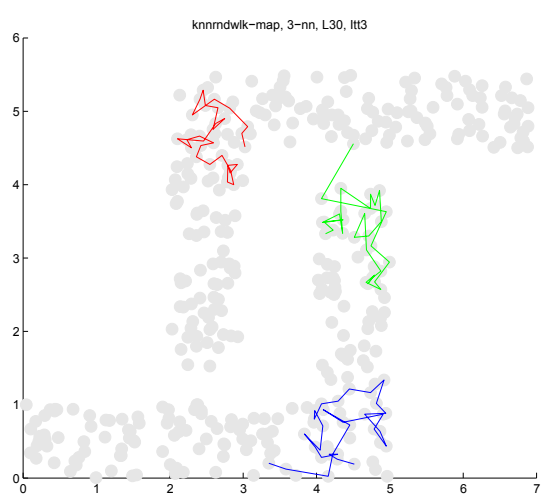

(a) 3 -nn, $\mathrm{L}=30, \mathrm{~S}=3$

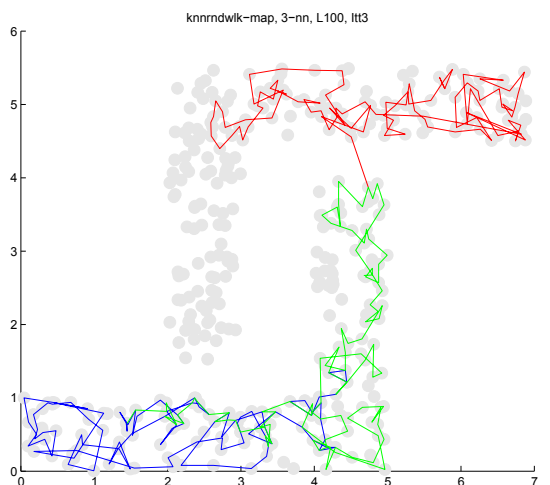

(d) $3-\mathrm{nn}, \mathrm{L}=100, \mathrm{~S}=3$

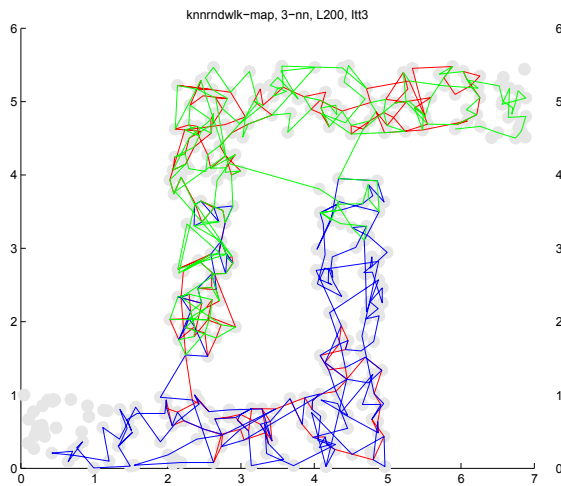

(g) $3-\mathrm{nn}, \mathrm{L}=200, \mathrm{~S}=3$

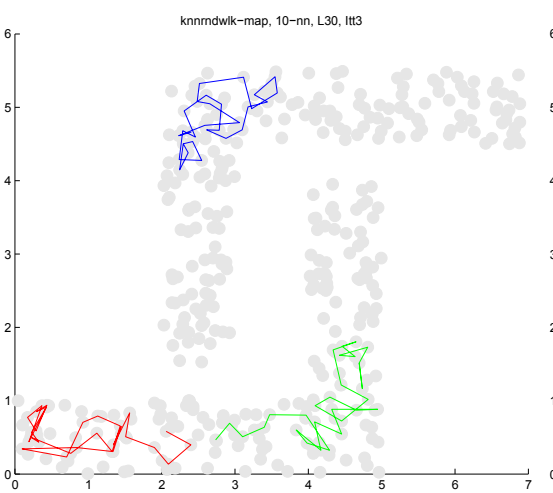

(b) $10-\mathrm{nn}, \mathrm{L}=30, \mathrm{~S}=3$

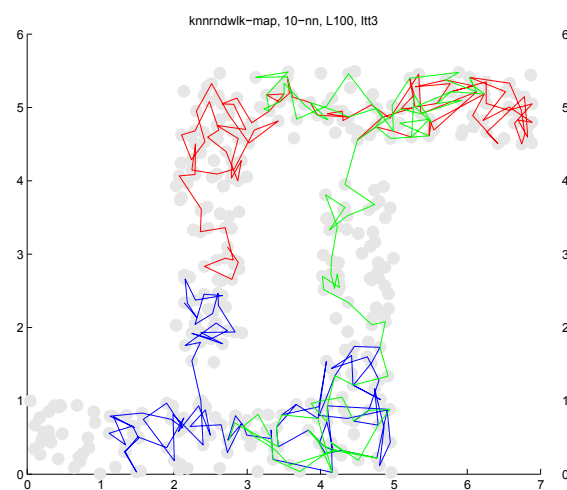

(e) $10-\mathrm{nn}, \mathrm{L}=100, \mathrm{~S}=3$

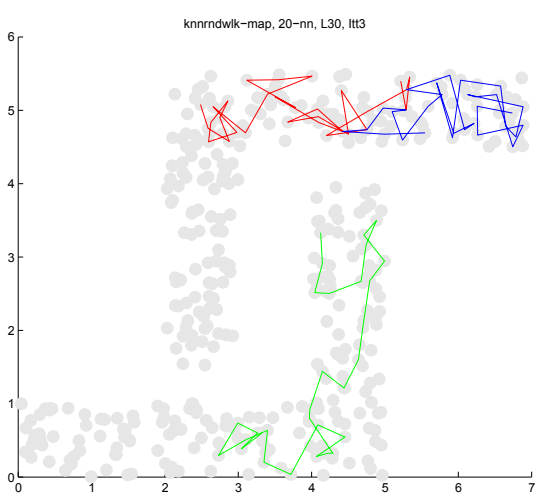

(c) $20-\mathrm{nn}, \mathrm{L}=30, \mathrm{~S}=3$

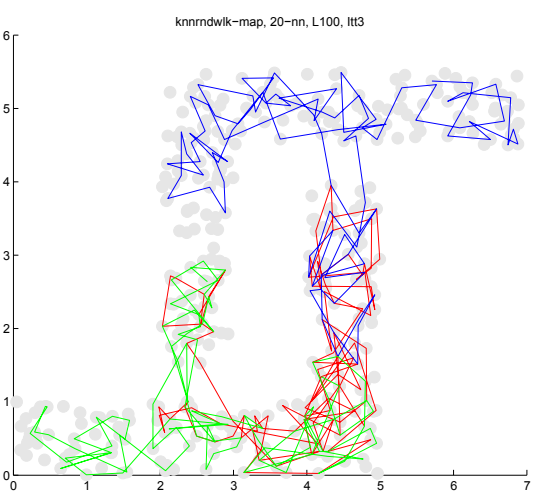

(f) $20-\mathrm{nn}, \mathrm{L}=100, \mathrm{~S}=3$

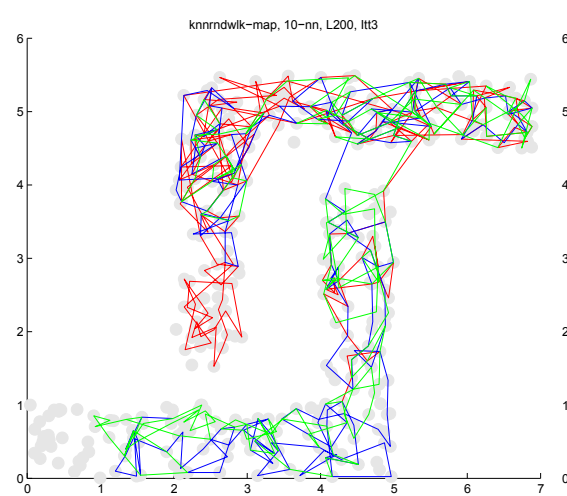

(h) $10-\mathrm{nn}, \mathrm{L}=200, \mathrm{~S}=3$

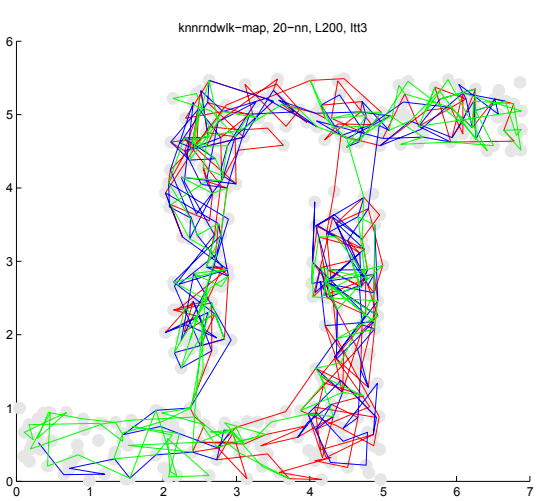

(i) $20-\mathrm{nn}, \mathrm{L}=200, \mathrm{~S}=3$

Figure 3 . The $k-\mathrm{nn}$ density walk map when $S=3$. 


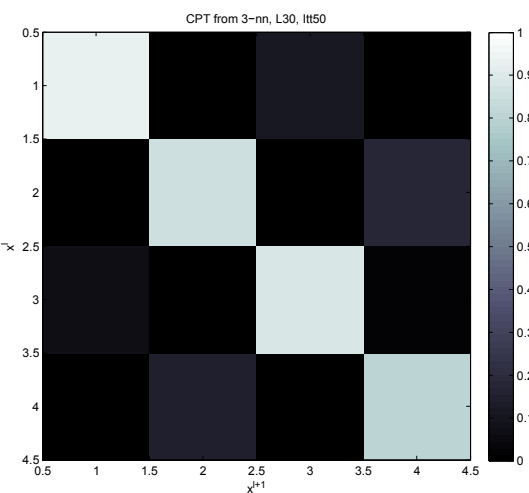

(a) 3-nn, $\mathrm{L}=30, \mathrm{~S}=50$

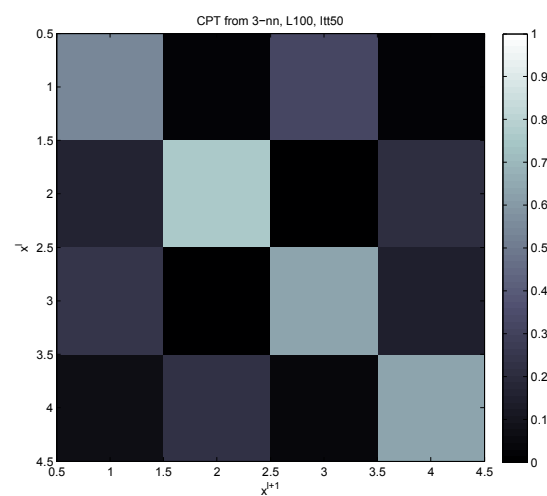

(d) 3-nn, L=100, S=50

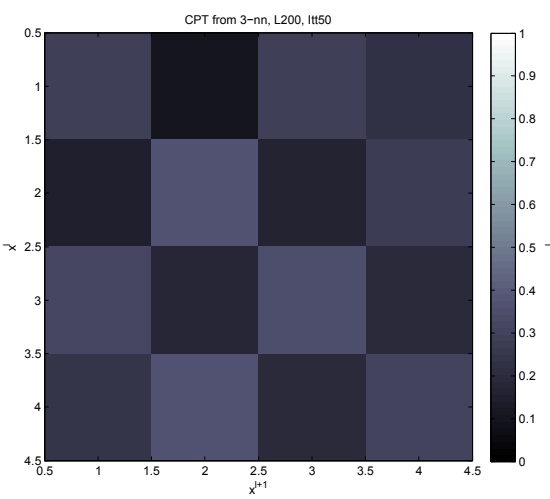

(g) 3-nn, L=200, S=50

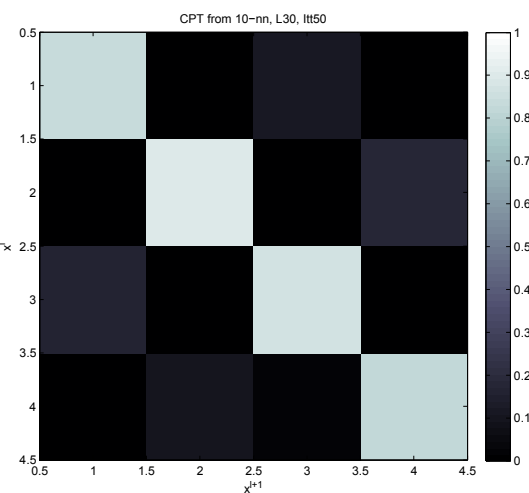

(b) $10-\mathrm{nn}, \mathrm{L}=30, \mathrm{~S}=50$

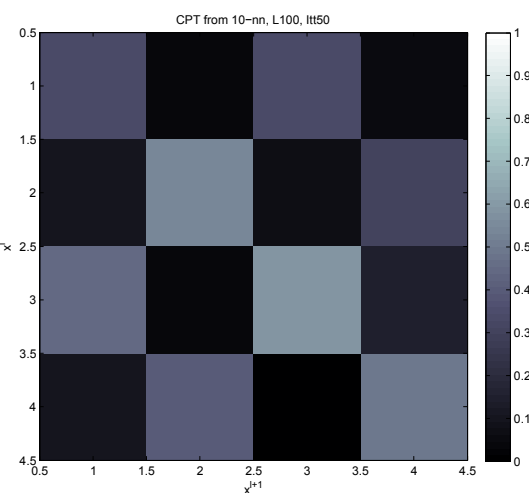

(e) 10-nn, L=100, S=50

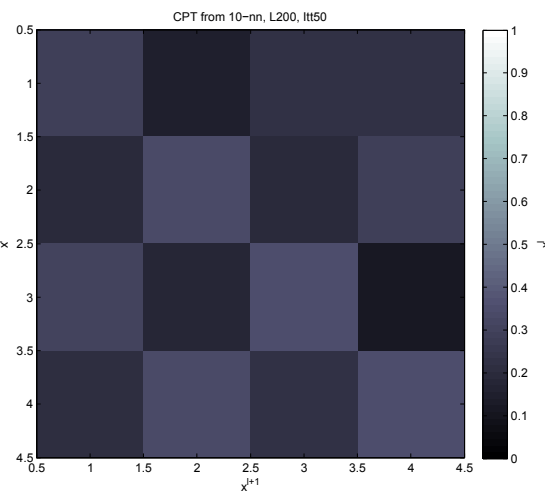

(h) $10-\mathrm{nn}, \mathrm{L}=200, \mathrm{~S}=50$

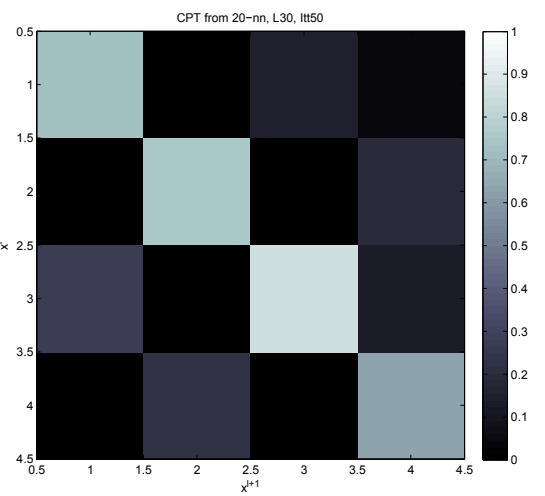

(c) 20 -nn, $\mathrm{L}=30, \mathrm{~S}=50$

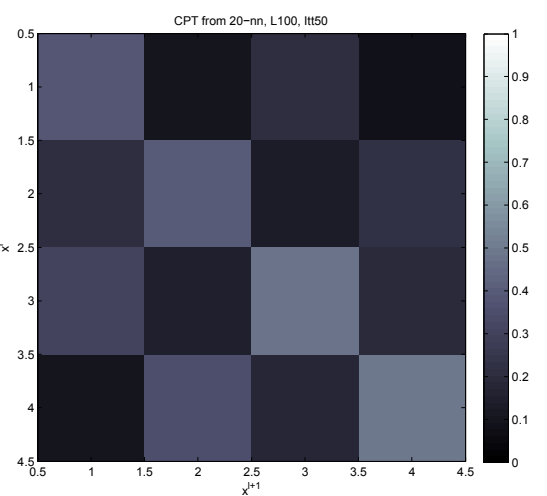

(f) $20-\mathrm{nn}, \mathrm{L}=100, \mathrm{~S}=50$

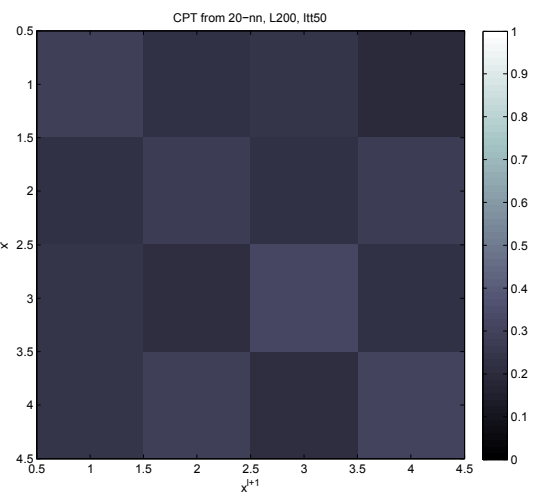

(i) $20-\mathrm{nn}, \mathrm{L}=200, \mathrm{~S}=50$

Figure 4. Resulting cpts from the KDW algorithm illustrated in Fig. 3. 
The numerator can be calculated efficiently using the sum-product algorithm ${ }^{14}$ which is a generalized case of belief propagation or Pearl's message passing algorithm, ${ }^{10}$ but the denominator requires summation over all possible structures $Z$ which might make the computation intractable when the number of nodes is large. There are some approximation techniques and heuristic optimization techniques to avoid the computational problem (e.g. simulated annealing and variational approximation ). One way to avoid the calculation of the denominator is to realize that the denominator is effectively a constant depending only on the observations $Y$, and consider the maximum a posteriori problem as an optimization problem where we don't have to explicitly evaluate the denominator or

which is equivalent to

$$
Z^{*}=\arg \max _{Z} P(Z \mid Y)
$$

$$
Z^{*}=\arg \max _{Z}\{\log P(Y \mid Z)+\log P(Z)\} .
$$

However, for simplicity we assume $P(Z)$ is uniformly distributed over $Z$, therefore we do not need to take $\log P(Z)$ into account when optimizing the structure. The likelihood of a structure $Z$ given the evidence $Y$ can be calculated from

$$
P(Y \mid Z)=\sum_{X} P(X, Y \mid Z),
$$

which can be evaluated exactly and efficiently using the sum-product algorithm since the structure of DEBAN is a tree. In this work we adopt a relatively simple simulated annealing optimization framework to solve for the best structure. The summary of the process can be found in section 5.1.

Once the optimal structure $Z^{*}$ is determined, we can gather the information at the root node $X_{r}$ of each tree from its corresponding leaf nodes via probabilistic inference:

$$
\begin{aligned}
P\left(x_{r} \mid Y, Z^{*}\right) & =\frac{P\left(x_{r}, Y \mid Z^{*}\right)}{P\left(Y \mid Z^{*}\right)}, \\
& =\frac{\sum_{X_{\mathcal{H}-\{r\}}} P\left(X, Y \mid Z^{*}\right)}{\sum_{X} P\left(X, Y \mid Z^{*}\right)},
\end{aligned}
$$

where $r$ is the index of a root node, and $X_{\mathcal{H}-\{r\}}$ denotes the set of all hidden variables except $x_{r}$. Note that the denominator in (8) is the same as (7), and the numerator of (8) can be obtained one step before (7) using the sum-product algorithm.

\section{EXPERIMENT AND RESULTS}

Before we present the experiments and results, we summarize the procedure used in the experiment and demonstrate the proposed structure updating algorithm.

\subsection{Summary of DEBAN for clustering}

The overview of DEBAN for unsupervised data clustering is summarized as follows:

1. Initialization

(a) Initialize $Z$ with the balanced-tree structure

(b) Initialize $\phi$ using KDW

(c) Initialize the parameters $\mu$ and $\Lambda$ with some appropriate values

2. Start simulated annealing routine using parameters in Table 1 to maximize $p(Y \mid Z)$ w.r.t. $Z$

3. Run the EM-DEBAN algorithm to re-estimate all the parameters $\mu, \Lambda$ and $\phi$ using Eqs. 4,5 and 6 respectively.

4. Calculate the likelihood of the given structure $Z, p(Y \mid Z)$ using sum-product algorithm.

5. Update the structure according to our proposed method given in section 5.2.

6. Repeat 3-5 in simulated annealing (SA) optimization framework, and get the optimal structure $Z^{\star}$.

8017 - 25 V. 1 (p.10 of 15) / Color: No / Format: Letter / Date: 2011-04-10 03:42:58 PM 
Table 1. SA parameters

\begin{tabular}{rl}
\hline \multicolumn{1}{c}{ parameters } & value \\
\hline initial temperature $T_{0}$ & 25 \\
temperature limit $T_{\text {limit }}$ & 0.05 \\
temperature schedule $\alpha_{T}$ & 0.9 \\
$k$ & 1 \\
max_accept_to_update & 20 \\
max_anneal_interval & 50 \\
max_iteration & 10000 \\
\hline
\end{tabular}

\subsection{Structure Updating Algorithm}

In this section, we propose a fast way to update the structure based on a stochastic method. Naturally, we want to connect a child node $x$ to a parent $P a(x)$ such that the state posterior marginals $p(x \mid Y)$ and $p(P a(x) \mid Y)$ are similar. Since the number of classes $C$ is the same from child level to parent level, the support of the posterior marginal distribution is the same for both layers. This facilitates us to use a simple distance measure of two distributions like the $L_{2}$-norm distance defined by

$$
D(f(x), g(x))=\sqrt{\sum_{i \in I}\left(f\left(x_{i}\right)-g\left(x_{i}\right)\right)^{2}}
$$

where $I$ is the support of the discrete distributions $f(x)$ and $g(x)$. For a child node $x_{j}$ and a parent node $x_{i}$ in the level $\mathcal{H}^{l}$ and $\mathcal{H}^{l+1}$ respectively, we calculate $D\left(p\left(x_{j} \mid Y\right), p\left(x_{i} \mid Y\right)\right)$ denoted by $D_{j i}$ for all $i \in \mathcal{H}^{l+1}$. We construct a multinomial distribution over the node index $i$ :

$$
\operatorname{Mult}(i \mid j)=\frac{\frac{1}{D_{j i}^{\alpha}}}{\sum_{i^{\prime} \in \mathcal{H}^{l+1}} \frac{1}{D_{j i^{\prime}}^{\alpha}}}
$$

where the random factor $\alpha$ determines the degree of the randomness when updating the structure. When $\alpha=0$, the structure updating algorithm becomes completely random algorithm, and when taking the limit of $\alpha$ to infinity, the algorithm becomes a stochastic greedy algorithm where the child node $x_{j}$ is connected to the parent $x_{i}$ with minimum distance. The choice of $\alpha$ depends on the data set and application, in our work we pick $\alpha=2$ so that we don't have to calculate the square root and because it can be interpreted as depending on the squared distances. The parent node $x_{i}$ that the child $x_{j}$ will be connected to in an iteration is determined stochastically by $i \sim \operatorname{Mult}(i \mid j)$, that is, sampling $i$ from the multinomial Mult $(i \mid j)$.

\subsection{Unsupervised Clustering using DEBAN}

The data set frame 2 comprises 2 classes, each of which is formed in an "L-shape" and has 200 points as shown in Fig. 5. It is obvious that each cluster cannot be modeled properly using a single Gaussian, so a GMM is used to model the data. We use Bayesian information criterion (BIC) to figure out the optimal number of the components for the GMM, which is 4, as shown in Fig. 5. The cpt $\phi$ is initialized using KDW parameters $k=3, L=100, S=50$ as shown in Fig. $6 f$. In order to test the robustness and for a reasonable run-time, the data set is downsampled from 400 to 32 samples, so the corresponding DEBAN, whose structure is initialized using a balanced tree, has 5 hidden levels and one observed level where the 32 observations are fed to. The state-observation distribution $p\left(y_{e} \mid x_{l}\right)$ is modeled using parameters $\mu$ and $\Lambda$ calculated from the GMM described earlier.

In the process, the state-transition cpt will be calculated locally using Eq. 6. To be more precise, each level $\mathcal{H}^{l}$ has its own cpt $\phi_{l v u}$ except for the top 3 levels. It is obvious that each of the top levels has very few nodes, therefore, it is a good idea to consider top 3 layers $(L-1$ to $L-3)$ to share the same $\operatorname{cpt} \phi_{(L-1) v u}$ (i.e. parameter tying). We follow the process given in Section 5.1, and the results are shown in Fig. 6.

\section{7 - 25 V. 1 (p.11 of 15) / Color: No / Format: Letter / Date: 2011-04-10 03:42:58 PM}




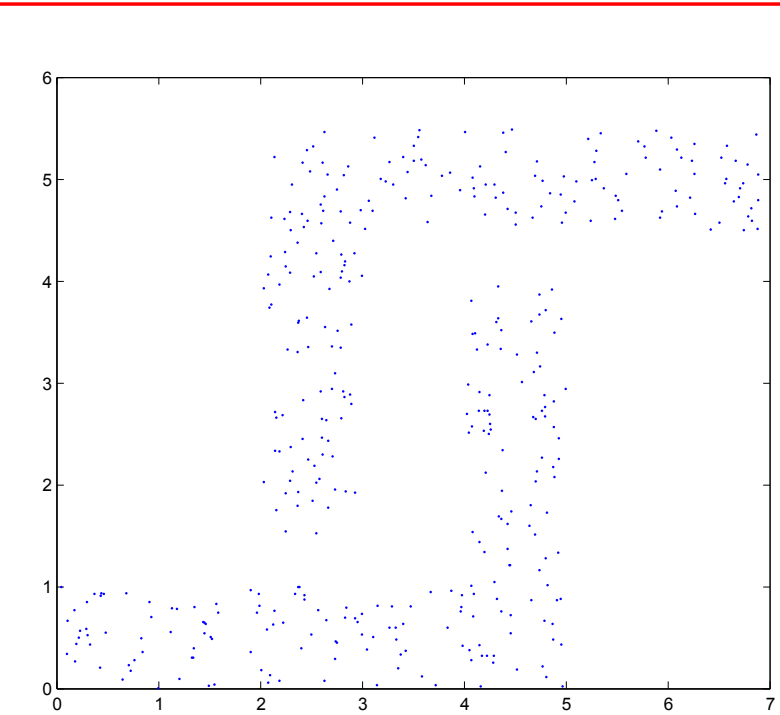

(a) observations

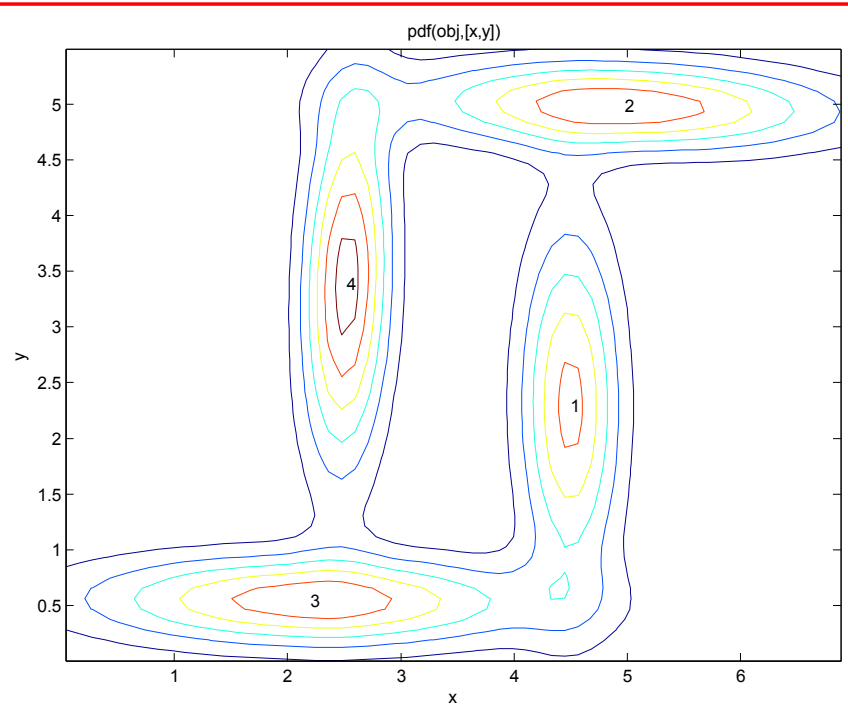

(b) Fit GMM to the observation

Figure 5. Dataset "frame2"

From the resulting clusters shown in Fig. 6 (a), the data samples are correctly separated into two clusters, each of which is the L-shape. The resulting optimal graph structure $Z^{*}$, shown in Fig. 6 (b), has two root nodes, each of which is corresponding to each of the L-shape cluster in Fig. 6 (a).

There are six rows in Fig. 6 (c), each of which, from top to bottom, represents the array of the final posterior distribution $p\left(x_{j} \mid Y\right)$ each node $x_{j}$ in root level $\left(H^{6}\right)$ to label level $\left(H^{1}\right)$ respectively. In each row, the x-axis and $\mathrm{y}$-axis represent the node number and the class respectively, and the magnitude of the probability ranges from black (0) to white (1). In level $\mathcal{H}^{1}$, there are 32 nodes (node \#32-\#63), and for simplicity we align samples from the same Gaussian component together, that is, the sample \#32-\#39 are from the same component; and \#40-\#47 are also from the same component and so on. The similarity between the distribution in level $\mathcal{H}^{l}$ and level $\mathcal{H}^{l+1}$ indicates that the child node and its corresponding parent tends to take the same state. Level $\mathcal{H}^{5}$ has a unique pattern of posterior which looks like the combination of its children. This is not a surprise because this event indicates the merging of two subclusters into the L-shape cluster.

In Fig. 6 (d), there are 4 matrices; the top $4 \times 4$ matrix represents the shared cpt of the level $\mathcal{H}^{4}$ to $\mathcal{H}^{6}$, the rest represent the cpt in level $\mathcal{H}^{3}$ to $\mathcal{H}^{1}$ from top to bottom respectively. As for each matrix, the states of child node and the parent node are denoted by row and column respectively. The cpts $\mathcal{H}^{3}$ to $\mathcal{H}^{1}$ have strong diagonal elements, meaning that children and corresponding parents are likely to have the same state. The interesting part is shown in the shared cpt of the level $\mathcal{H}^{4}$ to $\mathcal{H}^{6}$ because some off-diagonal elements get high values. In fact, this pattern encodes the information regarding which Gaussian components should be merged into L-shape cluster which can be associated with the posterior distribution in Fig. 6 (c). The learning curve in Fig. 6 (e) illustrates the iterations and its log-likelihood in $\mathrm{x}$ and $\mathrm{y}$-axis respectively. SA is guaranteed to converge to the global optimal solution when the annealing period is big enough, therefore, in this experiment, we have used a long annealing period as shown in Table 1, and the experiment takes 1.5 hours to finish on a desktop with an Intel Pentium D $2.8 \mathrm{GHz}$ CPU, $3.62 \mathrm{~GB}$ of RAM, and programmed in MATLAB R2010a. In fact, the curve shows that the algorithm yields a good solution in the early iterations, so a quicker solution is possible with a better SA annealing schedule.

\section{DISCUSSION}

Theoretically this framework can be viewed as a more generalized framework of GMM and HMM. In a GMM each state variable (or component label) $x_{j}$ is assumed independent of others, whereas DEBAN assumes conditional independences among those variables by putting a tree-structure prior over the state variables. In an HMM, the state variables are correlated by a Markov chain which is seen as 1-D special case of DEBAN. DEBAN also 


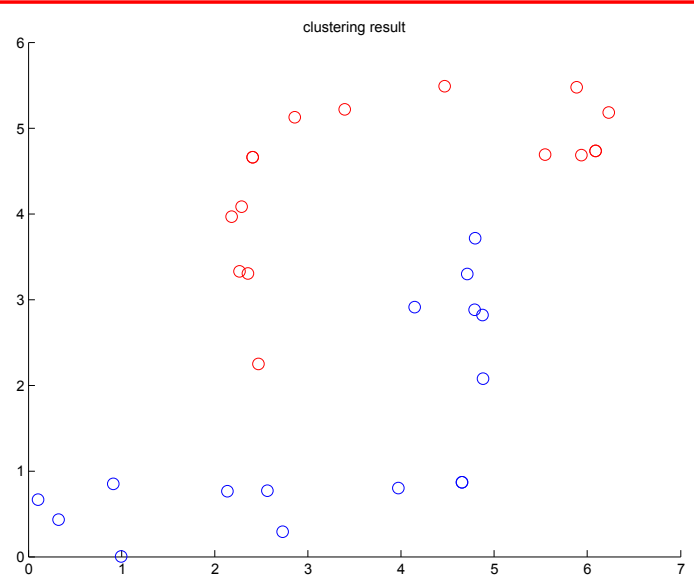

(a) Resulting clusters

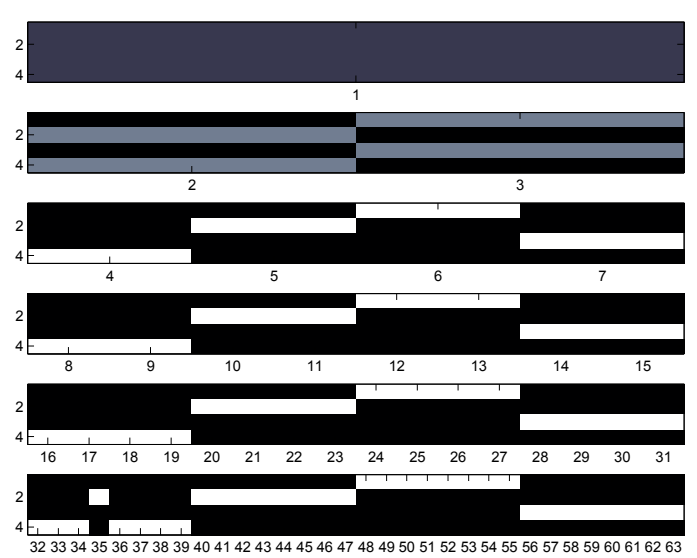

(c) Posterior distribution at each hidden node

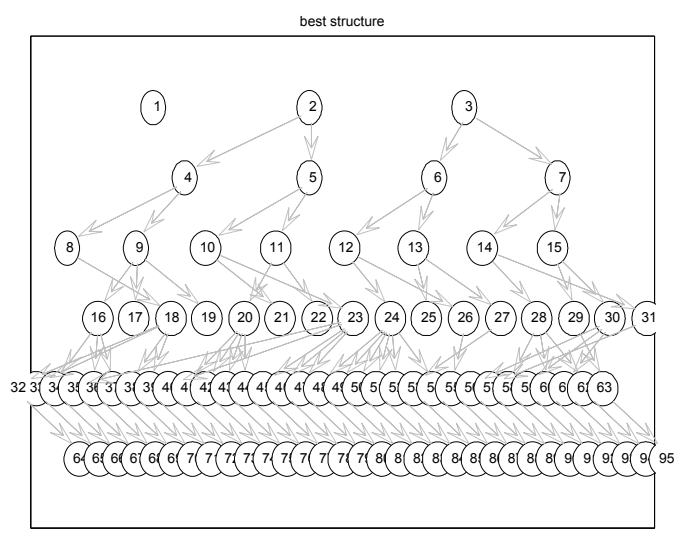

(b) The best structure from the optimization

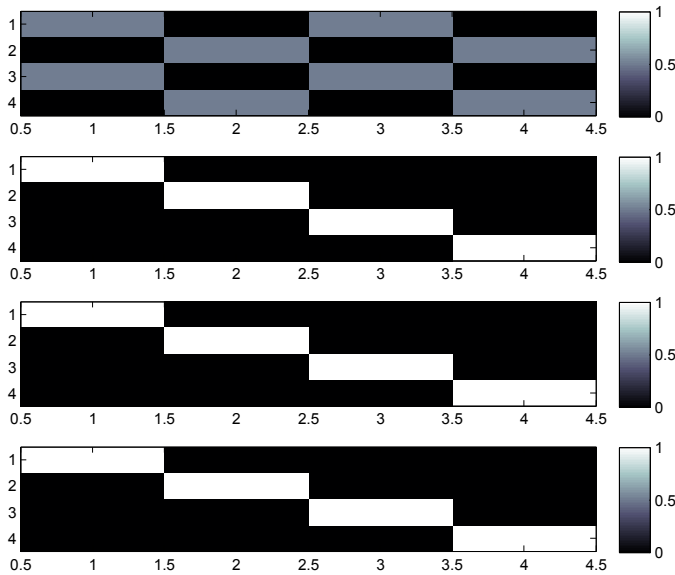

(d) The final cpt. From bottom to top $\phi_{(1) v u}$, $\phi_{(2) v u}, \phi_{(3) v u}$ and $\phi_{(4-6) v u}$ respectively.

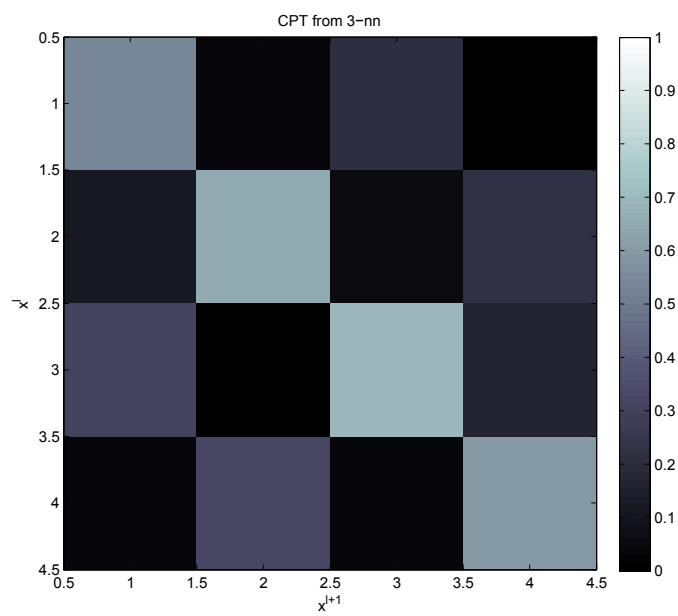

(f) The initial cpt for the whole model. (e) The learning curve; red and blue represent accepted and rejected respectively

Figure 6. DEBAN clustering results (a), the final tree structure (b), hidden node posterior distribution (c), final cpt entries (d), learning curve (e), and initial cpt (f). 
generalizes DT in the sense that it makes an obvious connection between state nodes and the observation nodes, and provides a framework to apply to other types of data besides images. There can be a lot more extensions to our proposed method, for instance, using a more complex model for the state-observation $p\left(y_{e} \mid x_{i}\right)$, using a different number of states for $x_{j}$ in different levels which makes more sense in the real-world problem, applying a Bayesian prior over the hidden variables, etc. DEBAN also includes intrinsically the ability to determine the number of clusters in the dataset accurately which is derived from the deformable graph structure $Z$. KDW demonstrates good results in initializing the cpt, but it is also interesting to use other kinds of graphs to initialize $\phi$. We are currently exploring a way to come up with a better annealing schedule such that the solution is found in an acceptable period of time. Also we are considering using approximate inference techniques in order to make the whole process faster.

\section{CONCLUSIONS}

We have developed DEBAN, a novel probabilistic framework based on a hierarchical tree-structured Bayesian network that is capable of adapting its graph structure to incorporate feature vectors at the leaf level. The multiscale nature of DEBAN enables us to discover (sub)structures in the data and dependence relationships among them. DEBAN yields a general framework which can be applied to any structured data in general. We have demonstrated that DEBAN performs unsupervised clustering well on a challenging dataset frame2 which cannot be modeled successfully using a GMM as each cluster is not a single Gaussian. Moreover, DEBAN solves for the appropriate number of cluster automatically from the data, this eliminates the need of having model selection explicitly. Together with DEBAN, we also propose the $k$-nn density walk as a powerful and fast way to initialize the state-transition cpt. In addition, we propose a stochastic greedy structure updating algorithm which yields good results. In future work we wish to find a better SA annealing schedule and to develop variational approximation algorithms such as mean-field variational approximation in order to speed up the algorithm run-time.

\section{ACKNOWLEDGMENTS}

This work was supported by the Office of Naval Research, Code 321OE.

\section{REFERENCES}

[1] Adams, N., Storkey, A., Ghahramini, Z., and Williams, C., "MFDTs: Mean field dynamic trees," in [Proc. 15th Int'l Conf. Pattern Recognition], 3, 147-150 (Sept. 2000).

[2] Adams, N. and Williams, C., "Dynamic trees for image modeling," Image and Vision Computing 21, 865877 (2003).

[3] Williams, C. and Adams, N., "DTs: Dynamic trees," in [Proc. Advances in Neural Information Processing Systems], Kearns, M., Solla, S., and Cohn, D., eds., 11 (1999).

[4] Storkey, A. and Williams, C., "Image modeling with position-encoded dynamic trees," 25(7), 859-871 (2003).

[5] Chow, C. I., Member, S., and Liu, C. N., "Approximating discrete probability distributions with dependence trees," IEEE Transactions on Information Theory 14, 462-467 (1968).

[6] Cooper, G. F. and Dietterich, T., "A bayesian method for the induction of probabilistic networks from data," in [Machine Learning], 309-347 (1992).

[7] Heckerman, D. and Chickering, D. M., "Learning bayesian networks: The combination of knowledge and statistical data," in [Machine Learning], 20-197 (1995).

[8] Marina Meila, M. I. J., "Learning with mixtures of trees," Journal of Machine Learning Research (2000).

[9] Feng, X., Williams, C., and Felderhof, S., "Combining belief networks and neural networks for scene segmentation," Pattern Analysis and Machine Intelligence, IEEE Transactions on 24, 467 -483 (Apr. 2002).

[10] Pearl, J., [Probabilistic Reasoning in Intelligent Systems: Networks of Plausible Inference], Morgan Kaufmann, San Francisco, CA (1988).

[11] Weiss, Y., "Correctness of local probability propagation in graphical models with loops," Neural Comput. 12(1), 1-41 (2000).

8017 - 25 V. 1 (p.14 of 15) / Color: No / Format: Letter / Date: 2011-04-10 03:42:58 PM 
[12] Dempster, A. P., Laird, N. M., and Rubin, D. B., "Maximum Likelihood from Incomplete Data via the EM Algorithm," Journal of the Royal Statistical Society. Series B (Methodological) 39(1), 1-38 (1977).

[13] Bilmes, J., "A Gentle Tutorial of the EM Algorithm and its Application to Parameter Estimation for Gaussian Mixture and Hidden Markov Models," (1998).

[14] F. R. Kschischang, B. J. F. and Loeliger, H. A., "Factor graphs and the sum-product algorithm," IEEE Transactions on Information Theory 47, 498-519 (2001). 